\title{
Curved Surfaces in Disordered Carbons by High Energy X-ray Scattering
}

\author{
A. Burian ${ }^{a, *}$ A. Szczygielska ${ }^{a}$, J. Kołoczek ${ }^{a}$, J.C. Dore ${ }^{b}$, \\ V. HONKIMAKI ${ }^{c}$ AND S. DUBER ${ }^{d}$ \\ ${ }^{a}$ A. Chełkowski Institute of Physics, University of Silesia \\ Uniwersytecka 4, 40-007 Katowice, Poland \\ ${ }^{b}$ Physics Laboratory, University of Kent, Canterbury CT2 7NR, U.K. \\ ${ }^{c}$ ESRF, BP 220, 38042 Grenoble, France
}

${ }^{d}$ Faculty of Earth Sciences, Interdepartmental Laboratory of Structural Research

University of Silesia, Będzińska 60, 41-200 Sosnowiec, Poland

\begin{abstract}
Disordered carbons prepared by slow pyrolysis of saccharose and anthracene and subsequent heat treatment at $1000^{\circ} \mathrm{C}$ and $2300^{\circ} \mathrm{C}$ have been studied by high energy X-ray diffraction. Computer simulations of the powder diffraction patterns of fullerenes, nanotubes and carbon models have been compared with the experimental data after conversion to real space via the Fourier transform. The presence of fullerene- and nanotube-like fragments with non-six membered rings in the investigated samples has been deduced by detailed analysis of the radial distribution functions of the saccharose- and anthracene-based carbons and related to resistance to graphitization of the saccharose-based carbons and to stability of the growing crystallites in the case of the anthracene-based carbons. The obtained results are compared to high resolution electron microscopy and Raman studies.
\end{abstract}

PACS numbers: 61.10.-i, 61.10.Eq, 61.46.+w

\section{Introduction}

Carbons produced by pyrolysis of organic precursors are generally divided into graphitizing or non-graphitizing ones [1]. The former are soft and less porous and can be easily transformed into graphite under thermal treatment. The latter are much harder, have a complicated porous structure and cannot, or can only with difficulty, be transformed into graphite, even at temperatures of $3000^{\circ} \mathrm{C}$ or higher.

${ }^{*}$ corresponding author; e-mail: burian@us.edu.pl 
The internal surface of graphitization carbons can be enhanced by mild oxidation with carbon dioxide or steam which makes them useful for a wide range of commercial applications. The various steps leading to graphitization have been revealed by heating pyrolytic carbons $[2,3]$. Below $800^{\circ} \mathrm{C}$ the basic structure units, consisting of about three graphitic layers, each of approximately $10-20 \AA$ in diameter, are formed. At this stage the basic structural units look the same for graphitizing and non-graphitizing carbons. These layers are stacked without spatial correlations forming the so-called turbostratic structure [4]. As temperature increases to about $1500^{\circ} \mathrm{C}$ the number of layers within the basic structural units increases with a lower degree of misorientation between them. Such behaviour is observed in graphitizing carbons and distinguishes them from non-graphitizing ones. At about $1900^{\circ} \mathrm{C}$ the columnar structure disappears and rippled layers are formed. Simultaneously, the in-plane and $c$-axis crystallites sizes rapidly increase. The graphitic structure develops then with increasing temperature. The graphitization temperature is usually reported to be $2300^{\circ} \mathrm{C}$. At this temperature most of the interplane structural defects are eliminated and the interlayer distance decreases. On the other hand the non-graphitizing carbons remain disordered at higher temperatures. However the increase in the basic structure units is observed.

Although pyrolytic carbons are produced for about fifty years, their atomic structure is not fully understood. The problem of different behaviour of graphitizing and non-graphitizing carbons upon heat treatment and their porous structure remain still unanswered (see the review paper by Harris [5]). Discoveries of fullerenes and the carbon nanotubes have prompted several authors to develop models for non-graphitizing carbons containing non-six-membered rings (e.g. pentagons or heptagons) besides the graphitic six-membered ring [5-8]. Recently, the presence of curved fullerene- and nanotube-like fragments in non-graphitizing carbons has been revealed by careful observation by high resolution transmission electron microscopy observation (HRTEM) [5, 8, 9] and by Raman scattering (RS) $[10,11]$. On the other hand, the high flexibility of graphene sheets explains their tendency to folding. Such folded sheets have been observed in a synthetic graphite prepared by arc evaporation [12] and by the pyrolysis of acenaphthylene [13]. Their presence can reduce the surface energy and therefore stabilize the structure of growing crystallites, as it has been deduced from Raman scattering studies $[10,11]$.

The purpose of the present work is to analyze carefully the high energy X-ray diffraction data to study the structure of saccharose- and anthracene-based carbons. These materials were chosen as two extreme examples of non-graphitizing (saccharose-based) and graphitizing (anthracene-based) carbons. The annealing temperatures $1000^{\circ} \mathrm{C}$ and $2300^{\circ} \mathrm{C}$ were selected because (i) pyrolytic carbon materials heat treated at $1000^{\circ} \mathrm{C}$ contain only pure carbon (within the $1 \%$ tolerance for contamination by hydrogen or oxygen) and (ii) the temperature of $2300^{\circ} \mathrm{C}$ is (as mentioned above) commonly considered as the graphitization temperature. 
The details of preparation of the carbon materials, studied in the present work, can be found elsewhere [11]. The X-ray diffraction data were recorded on the ID15A high energy beamline at European Synchrotron Radiation Facility (ESRF, Grenoble, France) in the range of the scattering vector $K=4 \pi \sin \theta / \lambda$ (2 $2 \theta$ is the scattering angle, $\lambda$ is the wavelength) from 0.5 to $24.0 \AA^{-1}$. The data processing was reported in detail in [10].

\section{Theoretical outline}

For disordered and polycrystalline materials the diffraction pattern arises from the coherent interference of waves scattered at distributed atoms being the scattering centres. In the case of identical atoms, for powdered samples, the intensity per atom averaged over all orientations in three-dimensional space can be written as

$$
I(K)=\frac{f^{2}(K)}{n} \sum_{i=1}^{n} \sum_{j=1}^{n} \frac{\sin \left(K r_{i j}\right)}{K r_{i j}} \exp \left(-\frac{K^{2} \sigma_{i j}^{2}}{2}\right),
$$

where $f(K)$ is the atomic scattering factor for carbon, $n$ denotes the number of atoms, $r_{i j}$ is the distance between $i$-th and $j$-th atom, $\sigma_{i j}$ is its standard deviation. The diffraction data can be converted to real space by the inverse Fourier transform yielding the reduced radial distribution function (RRDF) $d(r)$

$$
d(r)=4 \pi r\left[\rho(r)-\rho_{0}\right]=\frac{2}{\pi} \int_{0}^{K_{\max }} K \frac{I(K)-f^{2}(K)}{f^{2}(K)} \sin (K r) W(K) \mathrm{d} K,
$$

where $\rho_{0}$ is the number density describing the average number of atoms per unit volume (here per $\AA^{3}$ ), $W(K)$ is the window function which minimizes the truncation effects and $K_{\max }$ denotes the maximum value of the scattering vector attained in the experiment, limited by the wavelength. In the present work the following form of the window function was taken for computations $W(K)=$ $\sin \left(\pi K / K_{\max }\right) /\left(\pi K / K_{\max }\right)$. Assuming the three-dimensional Gaussian distribution of the interatomic distances, the radial distribution function (RDF) $4 \pi r^{2} \rho(r)$ can be expressed as the convolution of the true $4 \pi r \rho(r)$ function with the so-called "peak shape function" $P(r)$ given by Eq. (4) and then multiplied by $r$

$$
\begin{aligned}
& 4 \pi r^{2} \rho(r)=r \sum_{k} \int_{0}^{\infty} \frac{N_{k}}{\sqrt{2 \pi} \sigma_{k} r_{k}}\left\{\exp \left[-\frac{\left(r^{\prime}-r_{k}\right)^{2}}{2 \sigma_{k}^{2}}\right]-\exp \left[-\frac{\left(r^{\prime}+r_{k}\right)^{2}}{2 \sigma_{k}^{2}}\right]\right\} \\
& \quad \times\left[P\left(r-r^{\prime}\right)-P\left(r+r^{\prime}\right)\right] \mathrm{d} r^{\prime}
\end{aligned}
$$

where

$$
P(r)=\frac{1}{\pi} \int_{0}^{K_{\max }} W(K) \cos (K r) \mathrm{d} K
$$


$N_{k}$ is the number of atoms within $k$-th coordination sphere, $r_{k}$ is the radius of $k$-th coordination sphere. The sum in Eq. (3) is taken over all the coordination spheres. In the case of the turbostratic structure, in which single graphitic layers are stacked without spatial correlations along the $c$-axis, the RDF can be expressed as the sum of the RDF calculated for a single layer $(R D F)_{\mathrm{s} l}$ and the term related to averaged interlayer correlations ("the turbostratic term")

$$
\begin{aligned}
& 4 \pi r^{2} \rho(r)=(R D F)_{\mathrm{sl}} \\
& \quad+4 \pi r n_{\mathrm{a}}\left\{\frac{1-\exp \left(-d_{\mathrm{c}} / L_{\mathrm{c}}\right)^{\left[r / L_{\mathrm{c}}\right]}}{\exp \left(d_{\mathrm{c}} / L_{\mathrm{c}}\right)-1}-\frac{L_{\mathrm{c}}}{d_{\mathrm{c}}}\left[1-\exp \left(r / L_{\mathrm{c}}\right)\right]\right\},
\end{aligned}
$$

according to [14]. In Eq. (5) $n_{\mathrm{a}}$ is the number of atoms per unit area on a plane $\left(\rho_{0}=n_{\mathrm{a}} / d_{\mathrm{c}}\right), d_{\mathrm{c}}$ is the inter-layer spacing, $L_{\mathrm{c}}$ is the mean crystallite size along the $c$-axis direction and $\left[r / d_{c}\right]$ represents the greatest integer in $r / d_{c}$. Equations (2), (3), and (5) were used to compute the RRDFs for the models considered in the next section.

\section{Results and discussion}

\subsection{Non-graphitizing carbons}

It has been previously shown [14-16] that the experimental RDF for non-graphitizing carbons can be satisfactorily fitted using the turbostratic model with the values of the $\sigma$ parameter increasing proportionally to $\sqrt{r}$, according to the paracrystalline theory [17]. However, slight deviations of the coordination numbers from the values expected for a single graphitic sheet have been observed. In the present work these deviations are analyzed more profoundly in order to understand their origin. The experimental RRDF of carbon prepared from saccharose and heated at $1000^{\circ} \mathrm{C}$ is compared in Fig. 1 in the $r$-range from 3 to $8 \AA$ with computer

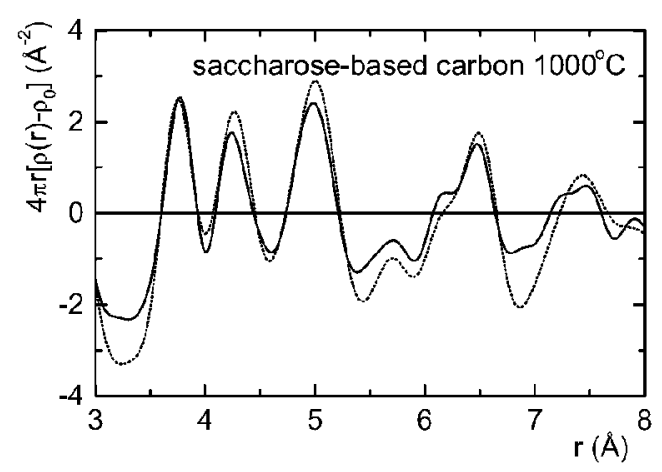

Fig. 1. The reduced radial distribution function of saccharose-based carbon heat treated at $1000^{\circ} \mathrm{C}$, experimental function (solid line) and the turbostratic simulation (dotted line). 


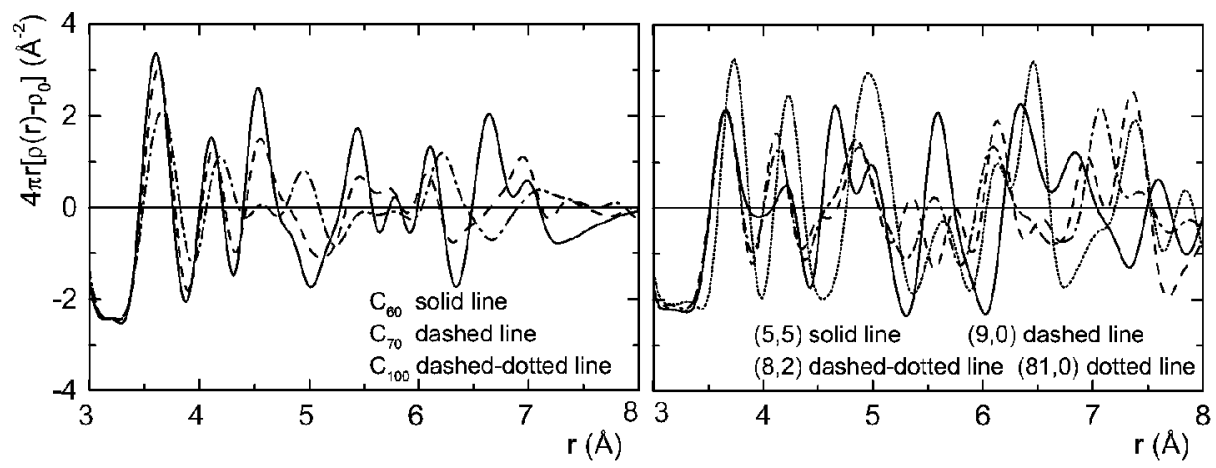

Fig. 2. The simulated reduced radial distribution functions of: the $\mathrm{C}_{60}$ (solid line), $\mathrm{C}_{70}$ (dashed line) and $\mathrm{C}_{100}$ (dashed-dotted line) fullerenes (left) and the $(5,5)$ (solid line), $(9,0)$ (dashed line), $(8,2)$ (dashed-dotted line) and $(81,0)$ (dotted line) nanotubes (right).

simulations based on the graphitic structure. In Fig. 2 the RRDFs computed for the $\mathrm{C}_{60}, \mathrm{C}_{70}$, and $\mathrm{C}_{100}$ fullerenes (left) and the $(5,5),(9,0),(8,2)$, and $(81,0)$ nanotubes (right) are shown for comparison. The Cartesian coordinates of the atoms which were used for computation of the nanotube model RRDFs were generated in the way described in [18] and for fullerenes were taken from [19]. The model for each of the $(5,5),(9,0),(8,2)$ nanotubes consists of about 100 atoms while the model of the $(81,0)$ nanotube contains 900 atoms. The meaning of the $(n, m)$ symbol, defining chirality of the nanotubes, are described in [20].

The 3-8 $\AA$ region was selected for the analysis because the first three peaks of the RRDFs, appearing at $r=1.41 \AA, 2.44 \AA$, and $2.84 \AA$ can be satisfactorily fitted within the precision of the coordination numbers of about $10 \%$ [16], assuming the structural parameters typical for the graphite structure. These peaks define hexagon - the basic structural unit. For saccharose-based carbon heated at $1000^{\circ} \mathrm{C}$ the turbostratic model consisting of three graphitic layers with the $\sigma$ values from $0.07 \AA$ at $r=1.41 \AA$ to $0.25 \AA$ at $r=12.00 \AA$ was considered. The number of layers was estimated on the basis of previous findings $[15,16]$. The simulation results are compared with the experimental data in Fig. 1. From inspection of Fig. 1 it can be concluded that although the positions of the RRDF peaks are reproduced, slight differences in the peak amplitudes between the model and the experiment remain. The two peaks of the experimental RRDF, appearing at about $4.2 \AA$ and $5.0 \AA$, have lower intensities when compared with the simulated function. In this region, the RRDFs of the C60, and C70 fullerenes, shown in Fig. 2 as the solid and dashed lines, respectively, exhibit negative contribution. Similar features exhibit also the $(5,5),(9,0)$, and $(8,2)$ nanotubes, which can be the source of discrepancies between the experimental data and the model-based simulations. Higher amplitudes of the experimental RRDF in the $r$ range from $5.2 \AA$ to $6.2 \AA$ can be explained by positive contributions of the $\mathrm{C}_{60}, \mathrm{C}_{70}$, and $\mathrm{C}_{100}$ fullerenes to the RRDFs, observed in this range in Fig. 2 (left) as well as of the $(5,5),(9,0)$, 
and $(8,2)$ nanotubes shown in the right part of Fig. 2. The observed discrepancy between the simulated and experimental RRDF at about $6.6-7.2 \AA$ can be related to the features of $(5,5),(9,0)$, and $(8,2)$ of the nanotubes RRDFs and the $\mathrm{C}_{60}$, $\mathrm{C}_{70}$, and $\mathrm{C}_{100}$ fullerenes.

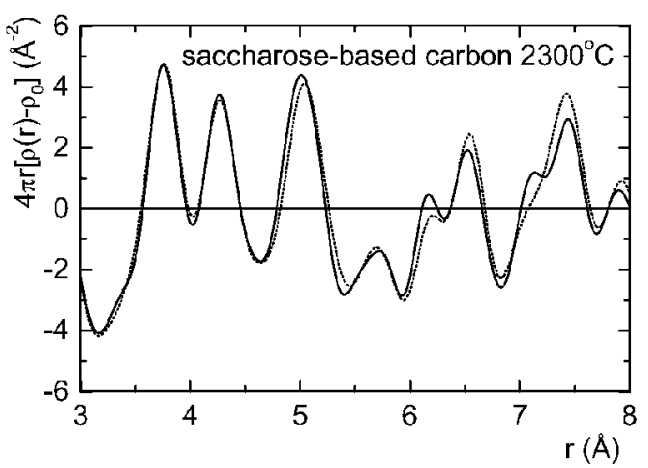

Fig. 3. The reduced radial distribution function of saccharose-based carbon heat treated at $2300^{\circ} \mathrm{C}$ (solid line) and the turbostratic simulation (dotted line).

At $2300^{\circ} \mathrm{C}$, the RRDF peaks seen in Fig. 3, are sharper (the peak width is described by the value of the $\sigma$ parameter) and have higher amplitudes. Such behaviour is due to an increase in size of the ordered regions $[10,11]$. The values of the $\sigma$ parameters ranging from $0.045 \AA$ at $r=1.41 \AA$ to $0.120 \AA$ at $r=$ $12.00 \AA$, according to the paracrystalline concept, were taken for computation for the saccharose-based carbon annealed at $2300^{\circ} \mathrm{C}$. It is important to notice that at $2300^{\circ} \mathrm{C}$ contribution to the diffraction pattern and the RRDF form ordered regions of the samples is more significant than that of much less ordered curved regions, which is expected to be relatively weaker. From the plots shown in Fig. 3 it can be seen that the graphite-based model does not fully match the experimental functions. A greater amplitude of the experimental peak at $5.0 \AA$ and its shift towards a lower $r$ value can be related to the peaks reported for all the nanotubes or for the C100 fullerene. These peaks may cancel out the negative contribution from the $\mathrm{C} 60$ and C70 fullerenes. Enhancement of the experimental peak at $6.2 \AA$ can be explained by the presence of the correlations typical for the fullerenes (Fig. 2 left) or for the $(9,0)$ and $(8,2)$ nanotubes (Fig. 2 right). The lowering of the experimental RRDF peak at about $7.5 \AA$ may be due to the negative part of the simulated RRDF for the fullerenes clearly seen in Fig. 2.

\subsection{Graphitizing carbons}

The results of the similar simulations, performed for the anthracene-based carbons, are shown in Figs. 4 and 5 for the samples heat treated at $1000^{\circ} \mathrm{C}$ and $2300^{\circ} \mathrm{C}$, respectively. Discrepancies between the experimental data and the results of model-based simulations can be seen in Figs. 4 and 5. The paracrystalline model 


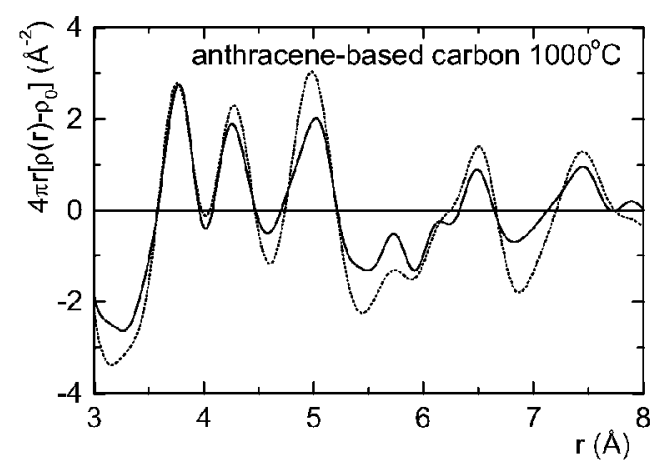

Fig. 4. The reduced radial distribution function of anthracene-based carbon heat treated at $1000^{\circ} \mathrm{C}$, experimental function (solid line) and the turbostratic simulation (dotted line).

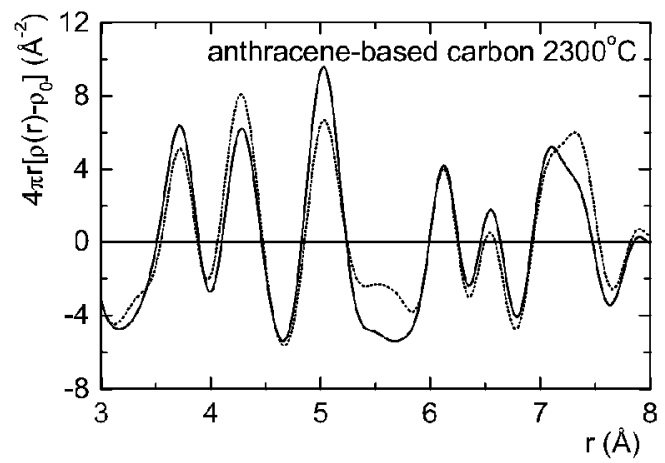

Fig. 5. The reduced radial distribution function of anthracene-based carbon heat treated at $2300^{\circ} \mathrm{C}$ (solid line) and the graphitic simulation (dotted line).

parameters $\sigma=0.045 \AA$ at $r=1.41 \AA$ and $\sigma=0.24 \AA$ at $r=12.0 \AA$ were taken for computation for the sample annealed at $1000^{\circ} \mathrm{C}$. At $2300^{\circ} \mathrm{C}$ the graphitization process is almost fulfilled for the sample prepared from anthracene $[10,11]$ and therefore the graphite model with $\sigma=0.04 \AA$ and $\sigma=0.12 \AA$ for intra and interlayer correlations was assumed to describe spatial correlations between atoms in the case of the anthracene-based carbon heated at $2300^{\circ} \mathrm{C}$. In the case of the anthracene based carbon differences between the experiment and the graphite simulation are rather related to the nanotube-like elements. Negative contributions from the $(5,5),(9,0)$, and $(8,2)$ nanotubes in the $r$ ranges $4.2-4.5 \AA$ and $5.0-5.2 \AA$ can lead to lower amplitudes of the experimental peaks at $4.2 \AA$ and $5.0 \AA$. Enhancement of the experimental RRDF in the region from $5.4 \AA$ to $6.0 \AA$ can be related to the $(5.5)$ and $(8,2)$ nanotube peaks observed at about $5.6 \AA$. Misfit at $6.6-7.2 \AA$ can be explained by the contribution of the $(5,5),(9,0)$, and $(8,2)$ nanotubes. At $2300^{\circ} \mathrm{C}$ the amplitudes of the experimental RRDF are appreciably higher the peaks are sharper. Higher amplitudes of the experimental peaks in com- 
parison with the graphite simulation at $3.7 \AA$ and $5.0 \AA$ are related to the peaks of the RRDF of the $(9,0),(8,2)$, and $(81,0)$ nanotubes. The $(5,5)$ nanotube was not considered as potential explanation of the differences between the experiment and the graphite-based simulation because of the relatively strong peak at $4.6 \AA$, which corresponds to the minimum of the experimental RRDF. The decrease of the highest of the peak at $4.2 \AA$ may be the result of the negative parts of the $(9,0)$ and $(8,2)$ RRDFs.

At this stage it should be pointed out that unambiguous distinction between fullerene- and nanotube-like components is not possible based on the diffraction data only. The contributions to the RRDF coming from the fullerenes and nanotubes shown in Fig. 2 exhibit different amplitudes which are out of phase in certain $r$ regions in comparison with the experimental RRDF. Moreover, the presence of perfect fullerenes or nanotubes is not expected in the case of carbons studied in the present work. Instead, the occurrence of the curved or curled elements is more likely. In the next section the present results are compared with those of Raman and electron microscopy studies.

\subsection{Relation to Raman and electron microscopy studies}

The conclusions drawn in the previous sections are in agreement with our studies carried out using HRTEM and RS [9-11]. In the case of non-graphitizing carbon (the saccharose-based carbon) at $1000^{\circ} \mathrm{C}$, curved elements or tightly curled carbon layers have been revealed by HRTEM [9]. Moreover, onion-like structure in which concentric sheets are curled around a central cavity and completely closed particles have been observed [8, 9]. HRTEM images have exhibited also fragments of the structure resembling nanotubes [8]. These findings are supported by the RS investigations $[10,11]$. On the other hand a typical HRTEM micrograph of the graphitizing carbon (the anthracene-based carbon) shows the structure in which the carbon layers are aligned along a preferred direction and packed closely together within the nanoscopic scale. These layers are curled but no closed cavities are observed. The curved fragments of the structure can affect the diffraction data in the similar way as the closed ones and can lead to discrepancies between the turbostratic model with the graphitic correlations within a single layer and the experimental data. Such conclusion is also in agreement with the RS studies for the graphitizing carbons $[10,11]$.

\section{Conclusions}

The high energy X-ray diffraction technique which utilizes the third generation synchrotron radiation source was used to characterize the atomic scale structure of the graphitizing and non-graphitizing carbons. This technique has proved to be an efficient tool for a detailed description of arrangement of atoms in the carbon materials studied. It was concluded that the faceting and curvature 
were due to the presence of pentagons or other non-hexagonal rings. However it is very difficult to conclude about the presence of the curves elements and their detailed structure based only on the diffraction technique as it has been suggested in our earlier papers $[14,15]$. The diffraction method should be regarded as the complementary technique for characterization of the structure of the carbon family, which together with HRTEM and RS can provide the experimental data for full understanding of the structure of microporous carbons at the atomic level.

\section{Acknowledgment}

A.B. and A.S. were financially supported by the State Committee for Scientific Research (Poland) grant No. 5 PO3B 11321.

\section{References}

[1] R.E. Franklin, Proc. R. Soc. Lond. A 209, 196 (1951).

[2] M. Guigon, A. Oberlin, G. Desarmot, Fibre Sci. Technol. 20, 55 (1984).

[3] M. Guigon, A. Oberlin, G. Desarmot, Fibre Sci. Technol. 20, 177 (1984).

[4] B.E. Warren, Phys. Rev. 59, 693 (1941).

[5] P.J.F. Harris, Int. Mater. Rev. 42, 206 (1997).

[6] S.J. Townsend, T.J. Lenosky, D.A. Muller, C.S. Nichols, V. Elser, Phys. Rev. Lett. 69, 921 (1992).

[7] H. Terrones, M. Terrones, W.K. Hsu, Chem. Soc. Rev. 24, 341 (1995).

[8] P.J.F. Harris, S.C. Tsang, Philos. Mag. A 76, 667 (1997).

[9] P.J.F. Harris, A. Burian, S. Duber, Philos. Mag. Lett. 80, 381 (2000).

[10] A. Burian, J.C. Dore, Acta Phys. Pol. A 98, 457 (2000).

[11] A. Burian, P. Daniel, S. Duber, J.C. Dore, Philos. Mag. B 81, 525 (2001).

[12] D. Ugarte, Chem. Phys. Lett. 198, 596 (1992).

[13] P.R. Buseck, H. Bo-Jun, L.P. Keller, Energy Fuels 1, 105 (1987).

[14] D.F.R. Mildner, J.M. Carpenter, in: Proc. 5th Intern. Conf. on Amorphous and Liquid Semicondictors, Garmisch Partenkirchen, 1973, Eds. J. Stuke, W. Brenning, Vol. 1, North-Holland, Amsterdam 1974, p. 463.

[15] A. Szczygielska, A. Burian, J.C. Dore, J. Phys., Condens. Matter 13, 5545 (2001).

[16] A. Szczygielska, A. Burian, S. Duber, J.C. Dore, V. Honkimaki, J. Alloys Comp., in press.

[17] A.M. Hindeleh, R. Hosemann, J. Macromol. Sci 34, 327 (1995).

[18] J. Kołoczek, Y.-K. Kwon, A. Burian, J. Alloys Comp., in press.

[19] http://sbchem.sunysb.edu/msl/fullerene.html.

[20] R. Saito, G. Dresselhaus, M.S. Dresselhaus, Physical Properties of Carbon Nanotubes, Imperial College Press, Singapore 1998, p. 35. 